\title{
A clinical pilot study on posture stabilization via light contact with cane-type companion robot
}

\author{
Shunki Itadera ${ }^{1 *}\left(\mathbb{0}\right.$, Tadayoshi Aoyama$^{1}$, Yasuhisa Hasegawa ${ }^{1}$, Keita Aimoto $^{2}$, Kenji Kato $^{2}$ and Izumi Kondo ${ }^{2}$
}

\begin{abstract}
In this paper, toward robotic gait assistance, we investigate the feasibility of a cane-type assistive mobile robot accompanying the user autonomously through a clinical pilot experiment. As widely known, gait ability is important for all people to keep their quality of life. However, for people having weakened lower limbs such as elderly people, their postural sway during walking could be insuppressible and cause falling. To support the gait motion of elderly people, our group has been developing a series of hand-holding cane robots named Intelligent Cane. Such assistive robots are expected to remove barriers to the independent lives of elderly people. Recently, we have focused on the potential of a companion robot that follows the user ahead and can be touched or grasped for bracing whenever the user needs it. In order to demonstrate proof of our concept through an experiment with a motion capture system, we propose a user companion strategy that enables our cane robot to keep a constant relative distance between the robot and the user walking on a treadmill. We evaluate the accuracy of the user companion in an experiment where a user walks on a treadmill. Then, we conduct a clinical experiment with three healthy subjects walking on with the treadmill with our cane robot as a pilot study. Through the clinical experiment, we evaluate a postural stabilization effect of physical interaction with the robot and discuss the feasibility of our robotic gait assistance methodology.
\end{abstract}

Keywords: Assistive robotics, Cane-type robot, Human-robot interaction, Companion robot

\section{Introduction}

Increasing elderly population and a shortage of human resources cause that the demand for care exceeds the supply [1]. The unbalanced relationship could lead to insufficient social security for elderly and excessive care burdens to caregivers [2]. As a solution to such a labor shortage, assistive robots are expected to support medical treatment and enhance physical ability more effectively [3]. Furthermore, the robotic devices which can be used without medical observation would help people with a wounded lower limb live independently.

Our goal in this study is to provide sufficient assistive robot interaction for gait stabilization and promote a daily locomotion habit. Demotivated and insufficient gait

\footnotetext{
*Correspondence: itadera@robo.mein.nagoya-u.ac.jp

${ }^{1}$ Department of Micro-Nano Mechanical Science and Engineering,

Nagoya University, Nagoya 464-8603, Japan

Full list of author information is available at the end of the article
}

activities have a possibility to be one of the dominant factors in disuse syndromes [4]. In addition, walking ability is closely related to our quality of life (QOL) [5]. Unbalanced gait puts elderly people at risk for falling and may cause a critical injury such as a bone fracture. Using a walking assistive robot, we address to enrich daily lives of elderly people through supporting gait locomotion.

As one of the approaches to stabilize gait movement, a number of research groups have previously addressed to develop wearable walking assistive robots, called exoskeletons, to rotate or constrain some joints of the user's whole body or lower limb (see [6,7] for reviews). Such exoskeletons are designed for elderly people who have difficulty in walking voluntarily without any assistance such as paraplegic patients. Exoskeletons are attached to the lower limbs of the user directly, and physically provide assistive torques to each user's joint in order to support knee flexion/extension and ankle plantarflexion/dorsiflexion. Notable examples of commercial exoskeleton 
devices include Lokomat by Hokoma [8], EksoGT by Ekso Bionics [9], Indego by Parker Hannifin Corp. [10], HAL by Cyberdyne [11] and WPAL by ASKA Corporation [12]. Research platforms for robotic gait assistance exoskeletons include ABLE [13] and EXPOS [14], whose objective is to improve mobility of elderly people. These assistive wearable robots are beneficial in increasing gait stability by assisting leg swing and preventing the joints of the lower limb from buckling due to insufficient muscle strength. Recently, the potential for such functionality has been evaluated in clinical tests with patients. However, the structure of the wearable device could restrict free lower limb movements of the users, and attachment mechanism should be carefully designed to be in contact with each patient individually in order to prevent skin issues such as skin wounds and pressure sores [7]. In addition, at the attachment and detachment of such a wearable robot, the therapists normally need to spend some time and labor, which would be time-consuming for the users and medical staff [15].

In order to support gait locomotion of elderly people with comfortable and straightforward usability, non-wearable assistive robots needing no attachment/ detachment have been explored. Typically, these robots are composed of a mobile base with a grip handle to provide walking support for elderly people by adjusting supportive forces, walking speeds and directions. These non-wearable robots do not constrain the leg movement of the user, and the walking ability of the elderly people can be evaluated in situations closer to the natural gait motions (unlike wearable systems with active forced assistance) using vital information. In [16], a device called intelligently controllable walker (i-Walker) has been introduced, which can analyze users' risk of falling with the force and position sensing information. In [17], a passive intelligent walker called RT-Walker has been proposed, which is composed of only passive elements such as servo-brakes to control the velocity of the walker according to the operation force applied by the user. In [18], a concept and implementations of the personal aid for mobility and monitoring (PAMM) system has been introduced, which provides guidance support for walking under adaptive shared control so that the user can intuitively control the robot. The primary focus of these studies is to support the user's walking in their living environment based on these robots' stiffness and mechanical stability.

In this study, we have proposed a notion of providing gait assistance considering human original stabilization ability as well as mechanical support. The related papers mentioned above present the assistive devices providing physical support to prevent the users' falling kinematically or dynamically. Although such a usual assistive strategy is useful to obtain a numerical balance margin, e.g., the zero moment point, our proposed notion focuses on human medical functionality to reduce the redundant interaction and help the user, who feels a fear to walk slightly, recovers more natural gait motion via light physical contact. Such a force propagation which is useful for posture stabilization has been discussed in the medical field. Light touch contact (LTC), which stands for lightly touching an object through a fingertip of a user, is known to have the potential to lead a hopeful physiological phenomenon that is strongly related to keeping balance [19]. An LTC with a static object is reported to be able to decrease postural sway while standing [20-22]. In addition, an LTC is useful to control postural sway caused by predictive and reactive disturbance [23]. The benefit of an LTC is also demonstrated in subjects with postural control dysfunctions, such as individuals with vestibular loss and peripheral sensory neuropathy [24, 25]. Effects of the LTC while walking on a flat floor and a treadmill are also reported [26, 27]. However, in these studies, equipped handrails are used as touched objects, and the subjects were instructed to stand or walk along with them. Recently, a mobile device that replaces such a touchable handrail is expected to investigate the touching effect in a living environment. In [28], the authors have developed a virtual LTC (VLTC) based wearable haptic device composed of an acceleration sensor and a vibration stimulator. By stimulating the user's fingertip according to its motion, the device provides an illusion that the user can lightly touch a virtual partition. Although the VLTC device has high usability, it could not support a user's weight physically in case of falling. In order to provide both the touching effect-based assistance and the mechanical assistance, we have addressed to develop a robot following the user ahead and providing a point which can be touched whenever the user requires.

In this paper, we propose a touchable robotic device accompanying the user automatically and supporting the user's weight in case of emergency, and investigate the feasibility of the robot in terms of the touching effect based postural stabilization through a clinical pilot experiment. Previously, our research group has been developing a series of cane-type robots named Intelligent Cane as a mobile hand-holding device $[29,30]$. With the focus on safe walking, we have introduced design strategies for an admittance control model for fall prevention of the user and robotic gait rehabilitation [31]. As another practical usage of our cane robot, recently, we have been exploring a robotic user companion system which enables the user to touch or grasp the cane robot whenever the user feels some necessity of some assistance during walking. We have designed a new prototype of the cane robot which is capable of providing the LTC effect based 
gait assistance mentioned above. In this paper, as a pilot study, we address to conduct a clinical experiment where subjects walk on a treadmill system including a motion capture in order to evaluate the touching effect from a view of the subjects' kinematics. For this experiment, we propose a motion planning strategy that enables the robot to move on the treadmill and follow the user. Then, through the pilot experiment, we evaluate the postural sway reduction based on the subjects' kinematic data and demonstrate a proof of our concept of human stabilization functionality based gait assistance using our canetype companion robot.

\section{Gait assistive robot "Intelligent Cane ver.6"}

This section presents an overview of a prototype of the cane-type robots named Intelligent Cane ver.6 proposed in this paper.

\section{Hardware description}

Figure 1 shows the main components of the cane robot whose purpose is to assist the user's mobility in daily life. This robot is designed to be capable of an active (accompanying) movement as well as a passive (compliance) control in living and hospital environments. The robot consists of (1) a caster drive mechanism-based mobile part, (2) a handle part with a free rotation joint, (3) a controller box with a mini PC, and (4) a sensor system including a 6-axis force/torque sensor, a laser range finder (LRF) and a web camera. The most notable feature of the robot is that its motion trajectory is planned according to the user's gait intention estimated based on the force applied to the handle and the user's leg movement measured by the force sensor and the LRF. By this trajectory planner, the cane robot can accompany a user and provide a contact point which can be touched whenever the user feels fears during walking. The cane robot is fully compatible with the robot operating system (ROS) [32].

We have designed this cane robot to satisfy the following requirements considering its daily use:

- Capable of supporting the user's weight while the user leaning on it.

- Movable at the speed of up to $1.3 \mathrm{~m} / \mathrm{s}$ which could be considered as a maximum walking speed of the elderly [33].

Figure 2 shows the system architecture of the cane robot. To keep the balance of the cane robot, its mobile base part is composed of two wheels and two omni-wheels. These normal wheels are actuated by $60 \mathrm{~W}$ DC motors (RE30-GB60, Maxon Motor), and omni-wheels are nonactuated, i.e. can be rotated freely according to external torques. The handle part has a variable-length stick and a free joint located just below the handle. Combining 2 degrees of freedom (DoF) of the mobile base part and 1 DoF of the free joint, we can achieve a quasi-holonomic handle positioning referring to the caster drive mechanism [34]. The controller box consists of a mini-PC (Intel

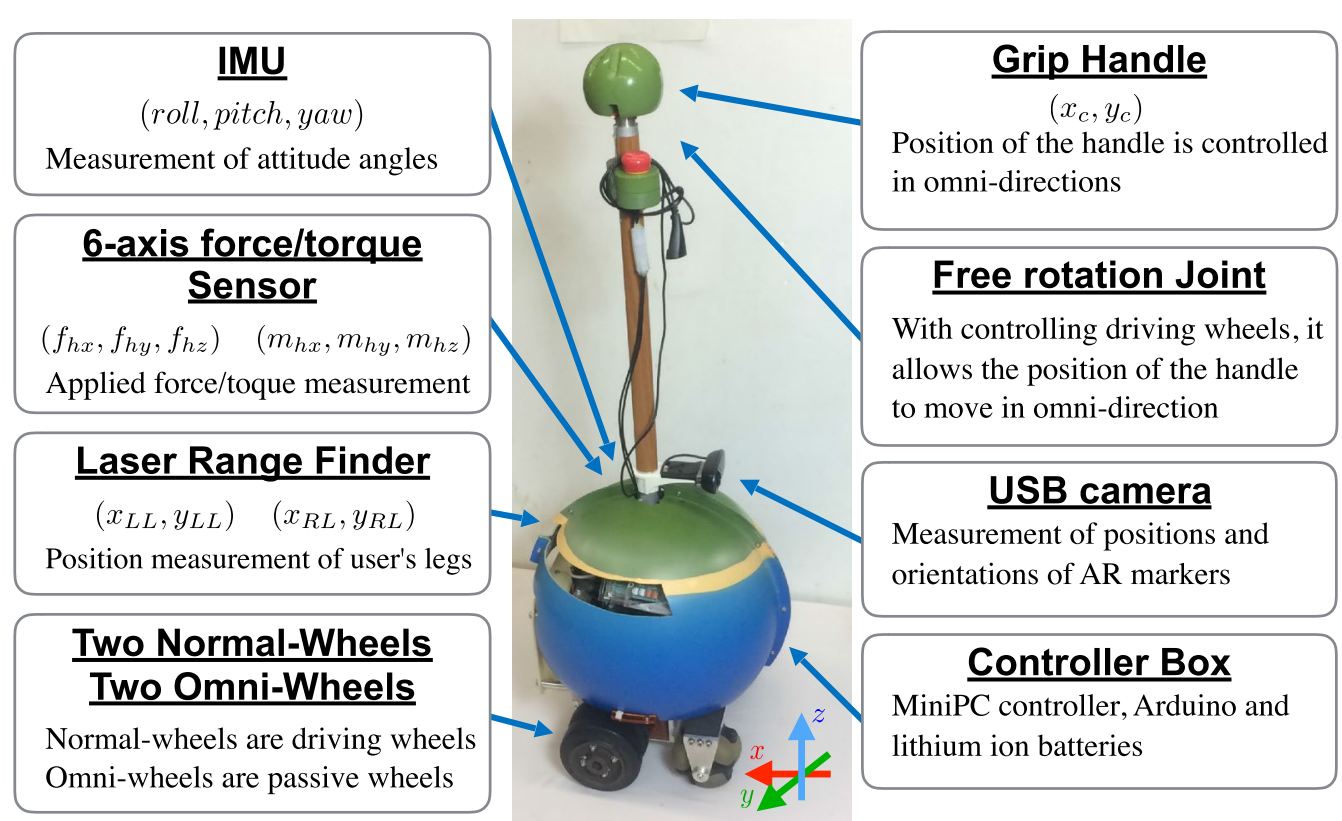

Fig. 1 Intelligent Cane ver.6. Components description of our cane-type robot used in this paper. This robot is designed to be capable of active movement, e.g., user companion 


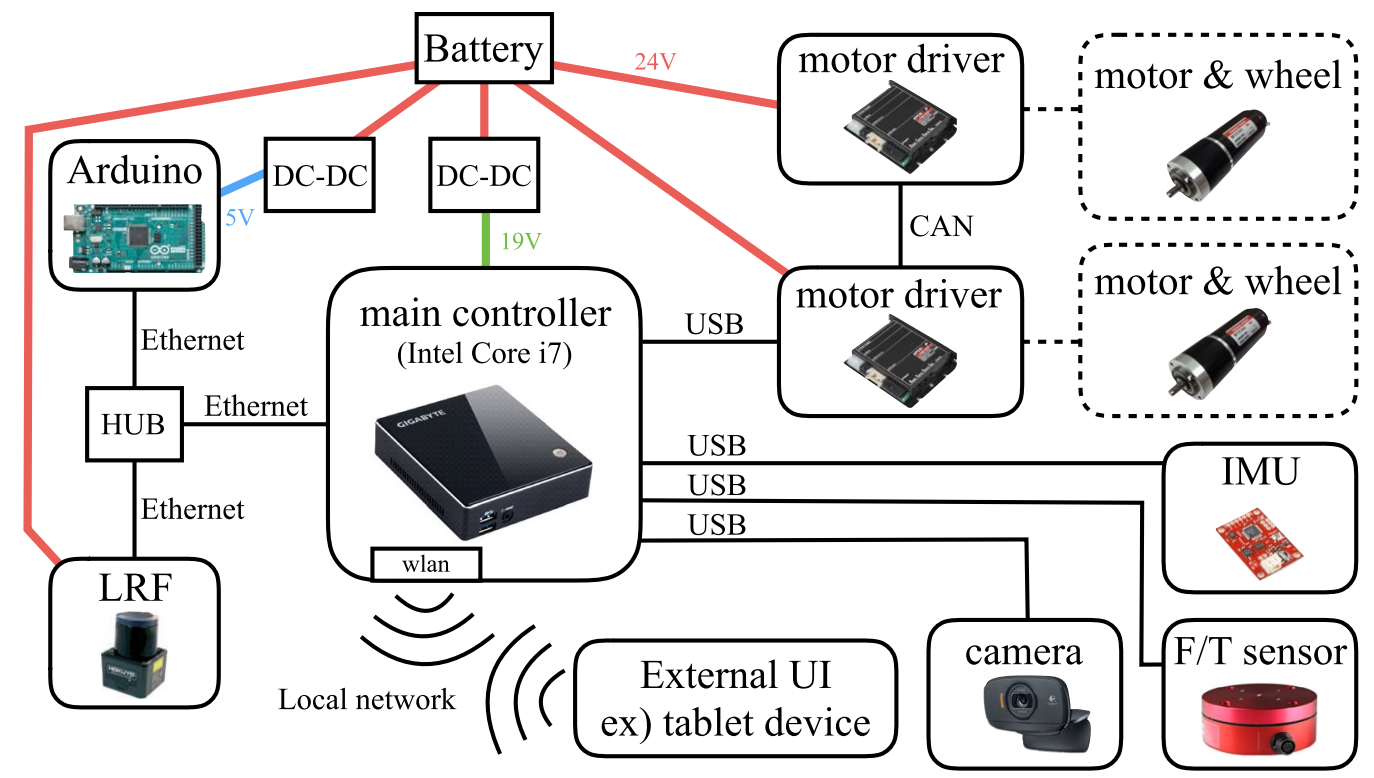

Fig. 2 Architecture of our cane robot. System structure of our cane robot including servo motors, a controller, and sensors

Core i7), an Arduino board and a lithium-ion battery. The mini-PC is used as a robot controller, and the Arduino board provides analog signal communication with a treadmill system mentioned below. The 6-axis force/ torque sensor (Dynpick WEF-6A500-10-RC5, WACOH THECH Inc.) is mounted between the mobile base and the cane stick and measures an interaction force applied to the robot by the user. The LRF (UST-10LX, HOKUYO AUTOMATIC CO., LTD) is mounted at a height of 20 $\mathrm{cm}$ above the ground and is used to detect the relative positions between the cane robot and the user's legs. The USB web-camera is fixed on the front side of the cane robot and used for self-localization by capturing AR markers in this paper.

\section{User interface}

Intuitive user interface (UI) is important for instructors who are not familiar with robotics such as medical doctors and physical therapists. We design the graphical and auditory UIs to improve the usability of the cane robot.

We design the useful web-based graphical user interface (GUI) to operate and monitor the cane robot, which is realized using roswww and rosbridge_server ROS package (see Fig. 3). This GUI can be accessed from a general web browser on a portable device connecting to the local network which the cane robot constructs automatically. Using this interface, the user can send operation commands to the cane robot intuitively. In addition, the GUI can be used to obtain the current user state such as the step length using the leg motion detection as well as the robot information. This method is available regardless of complicated configure for a client device.

In addition to the GUI, we develop an auditory user interface for the cane robot in order to take simplified communication with a user. At the beginning and the end of a gait trial, the robot speak specific sentences, e.g., "Let's walk with me!" in Japanese. In our previous research [30], we have reported that such an auditory communication has the potential to provide an affinity for the robot. This functionality is useful for the instructors as well to know the robot state without having a look at the above GUI and to concentrate on supporting the user physically during gait trials.

\section{User companion on treadmill}

This section presents the proposed self-localization and motion planning algorithm of our cane robot for user companion during treadmill walking.

\section{Self-localization}

Firstly, we explore a way to obtain the self-localization of the cane robot on the treadmill. The cane robot needs to detect its location accurately because incorrect self-localization may cause a collision accident due to the limited treadmill walking space. Although the original sensory system of the cane robot includes an IMU, which is generally used for the self-localization on a flat floor, such an inertial information-based sensor could not measure the motion of the robot running on the treadmill. To estimate the position of the cane robot in the world coordination fixed outside of 


\section{Inteligent Cane ver.6 Controller}

developed by Hasegawa Lab., Nagoya University

\section{Cane Robot Controller}

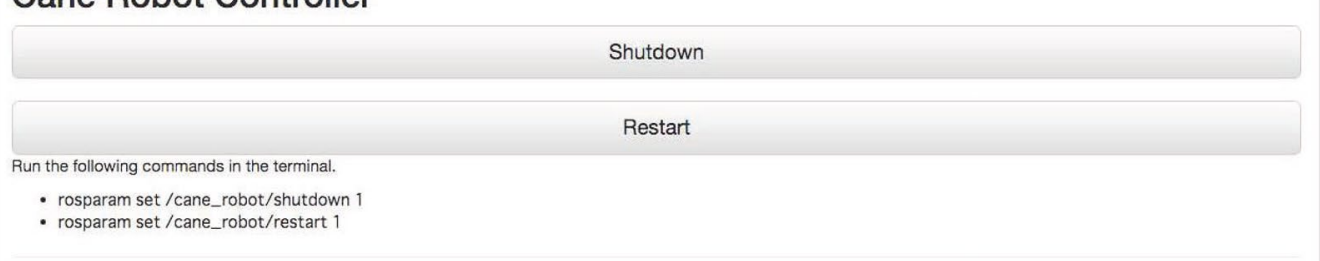

\section{Cane Robot Monitor}

contact force (/force/filtered)

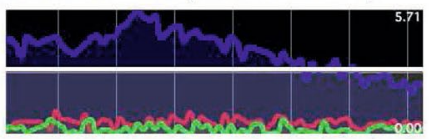

$\triangle$ :Force Touch状態です。閾值は3Nです。

\section{$+0.5 \mathrm{~N}$}

$-0.5 \mathrm{~N}$

Fig. 3 Graphical user interface of the cane robot. The developed GUI allows the user and the instructor to operate and monitor the cane robot intuitively. In the current implementation, they can send several commands such as "start" and "stop" and check the interaction force applied to the handle in real-time

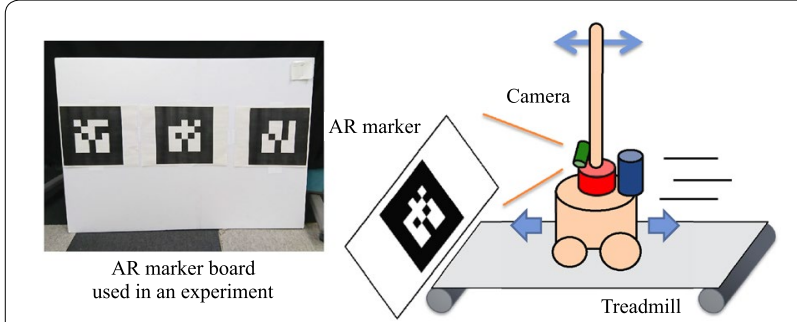

Fig. 4 AR markers usage in the experimental condition. An environment with AR marker where the robot moves on the treadmill (right), and AR marker board used in this experiment (left)

the treadmill, we place AR markers whose pose can be detected based on a camera image and their predefined size in front of the treadmill. The right figure in Fig. 4 illustrates the situation where the cane robot is running on the treadmill. To improve the robustness of the marker detection, we put three markers on a horizontal line, and the AR marker board used in this paper is shown in the left figure in Fig. 4. The relative position and orientation of the robot from the marker board can be calculated based on the average of three detected maker poses. Figure 5 shows the relative coordination with respect to the detected landmark pose.

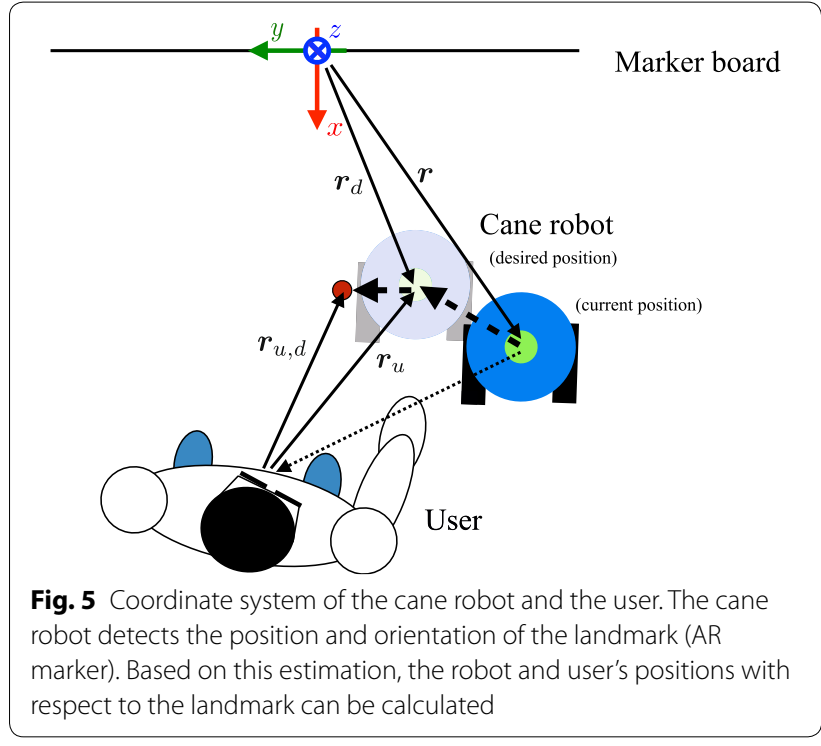

Note that, in this paper, we use the board where the AR markers are pasted and put in front of the treadmill. For better usability, we are planning to project the markers onto a screen where the user can see a virtual walking scene. Besides, we are looking into replacing 
the markers with another thing to make the landmark more natural.

\section{Motion control}

We present a motion control algorithm for autonomous user companion on the treadmill through physical and non-physical human-robot interaction. The velocity of the cane robot $v$ is determined according to PID control as follows:

$$
\boldsymbol{v}=\boldsymbol{K}_{p}\left(\boldsymbol{r}_{d}-\boldsymbol{r}\right)+\boldsymbol{K}_{i} \int\left(\boldsymbol{r}_{d}-\boldsymbol{r}\right) d t+\boldsymbol{K}_{d}\left(\dot{\boldsymbol{r}}_{d}-\dot{\boldsymbol{r}}\right),
$$

where $\boldsymbol{r}=[x y]^{T}$ is the vector of the cane robot position, $\boldsymbol{r}_{d}=\left[\begin{array}{ll}x_{d} & y_{d}\end{array}\right]^{T}$ is the vector of the desired position of the cane robot. Their coordinate system is shown in Fig. 5. $\boldsymbol{K}_{p}, \boldsymbol{K}_{i}$ and $\boldsymbol{K}_{d}$ are the diagonal matrix composed of PID gains in each direction. As can be seen from this equation, the specific motion can be achieved by updating the desired position $\boldsymbol{r}_{d}$ appropriately. We introduce the following two update rules:

1. Manual update according to the operation force applied by the user, and

2. Autonomous update to keep the selected relative position between the user and the cane robot.

The robot switches these rules according to the absolute of the applied interaction force $\boldsymbol{f}=\left[f_{x} f_{y}\right]^{T}$. If one of the components of the force vector exceeds a threshold $f_{\text {th }}\left(\left|f_{x}\right|>f_{\text {th }}\right.$ or $\left.\left|f_{y}\right|>f_{\text {th }}\right)$, the robot chooses the 1st rule (interaction force based manual update). In the other case $\left(\left|f_{x}\right| \leq f_{\text {th }}\right.$ and $\left.\left|f_{y}\right| \leq f_{\text {th }}\right)$, the robot follow the 2nd rule (relative position based autonomous update). The detailed update equations are described below.

\section{Interaction force based manual update}

In this rule, the user can control the robot position manually by applying suitable force directly, and move it to easily touchable location. The time variation of the desired robot position is defined by

$$
\dot{\boldsymbol{r}}_{d}=\boldsymbol{K}_{f} \cdot \boldsymbol{f}
$$

where $\boldsymbol{K}_{f}$ is the diagonal matrix composed of positive gain values in each direction. Following this rule, the robot can be operated by the user intuitively through such physical interaction.

\section{Relative position based autonomous update}

In this rule, the cane robot generates a trajectory to keep the relative distance to the user. We have developed the user position detector based on an open source ROS package leg_detector which can estimate the possible leg positions using LRF data [35]. Using the detected user's motion, the update rule is defined as follows:

$$
\dot{\boldsymbol{r}}_{d}=\boldsymbol{K}_{u, p}\left(\boldsymbol{r}_{u, d}-\boldsymbol{r}_{u}\right)+\boldsymbol{K}_{u, d}\left(\dot{\boldsymbol{r}}_{u, d}-\dot{\boldsymbol{r}}_{u}\right),
$$

where $\boldsymbol{r}_{u}$ is the vector of the relative position between the user and the cane robot and $\boldsymbol{r}_{u, d}$ is the desired relative position between them (see Fig. 5). $\boldsymbol{K}_{u, p}$ and $\boldsymbol{K}_{u, d}$ are the diagonal matrixes composed of gains of the PD controller. The desired relative position $\boldsymbol{r}_{u, d}$ is determined when the update rule is switched from (1) the manual update to (2) the autonomous update and kept constant while following the 2 nd update rule. This rule makes the robot move to keep the relative position defined by the user's intention.

\section{Safety limitation}

As mentioned above, the space on the treadmill where the user can walk is restricted, and the accompanying position should be in the limited space for the safety. Therefore we employ the simple limitation against the desired cane robot position $\boldsymbol{r}_{d}$ in (1). In the following passage, the desired position is limited as follows:

$$
x_{d, \text { min }} \leq x_{d} \leq x_{d, \text { max }}, y_{d, \text { min }} \leq y_{d} \leq y_{d, \text { max }},
$$

where $(*)_{d, \min }$ and $(*)_{d, \max }$ are minimum and maximum limitations in each axis, respectively, and they are defined according to the treadmill specification (mentioned below). In addition, we also employ the velocity limitation for the cane robot velocity $v$ in Eq. (1) to prevent any unintended motion.

\section{User companion test}

This section presents an evaluation of the proposed user companion algorithm, especially the 2nd rule: autonomous update to keep a constant relative distance between the user and the cane robot.

\section{Treadmill platform}

As a treadmill system platform, this study uses GRAIL (Motekforce, see [36]) at the National Center for Geriatrics and Gerontology, Japan. The system consists of (1) a 3D motion capture system, (2) a motorized fully instrumented treadmill, (3) a force plate, and (4) a cylindrical screen. Mainly using (1) the motion capture system, we capture the user's kinematic motion at a frequency of $100 \mathrm{~Hz}$.

In order to synchronize the motion data measured by GRAIL and the cane robot data, the robot sends trigger signals every $10 \mathrm{~s}$ and vertical force data through an analog signal connected to the GRAIL. These signals are recorded by the GRAIL system at a frequency of $100 \mathrm{~Hz}$. In addition, considering the size of the used treadmill, we 
set the desired position limitation of the cane robot in (4).

\section{Condition}

In this test, a user walks on the treadmill without touching the robot and move backward and forward. We measure the scrum position of the user and the center position of the cane robot using the motion capture system for a duration of $30 \mathrm{~s}$ and evaluate the accuracy of the proposed user companion algorithm. The coordinate system is shown in Fig. 6.

\section{Result}

Figure 7 shows the sacrum trajectory of the user in $\mathrm{x}$ - and $\mathrm{y}$-axes. The user walked approximately $0.2 \mathrm{~m}$ forward (from $t=10$ until $t=15$ ), and $0.3 \mathrm{~m}$ backward (from $t=20$ until $t=25)$. The snapshots $(t=5,15,25)$ are shown in Fig. 8. In addition, the bar plots of the mean and the standard division of the relative position between the user and the cane robot are shown in Fig. 9. Although the user moved about $0.5 \mathrm{~m}$ in $\mathrm{y}$-axis in the test, the standard division of the relative position was $0.075 \mathrm{~m}$. They indicate that the robot could absorb the user's movement to provide a contact point that can be touched by a walking user using our proposed user companion algorithm. On the other hand, a time delay for approximately $2 \mathrm{~s}$

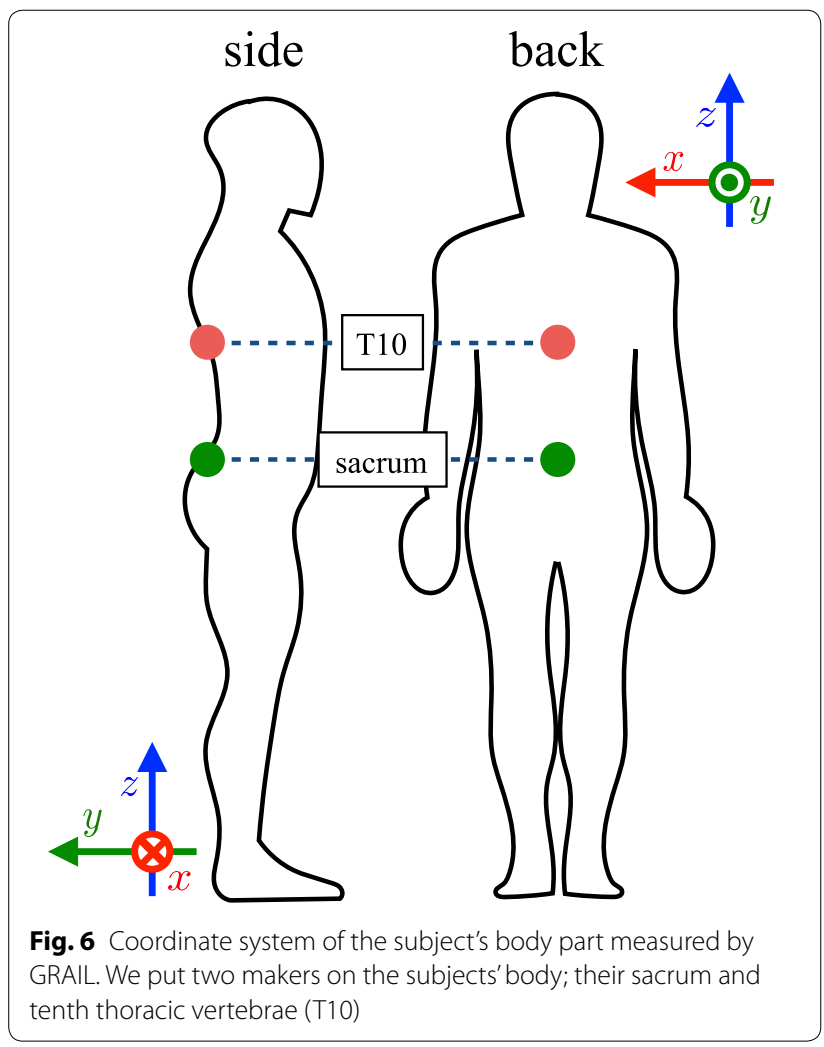

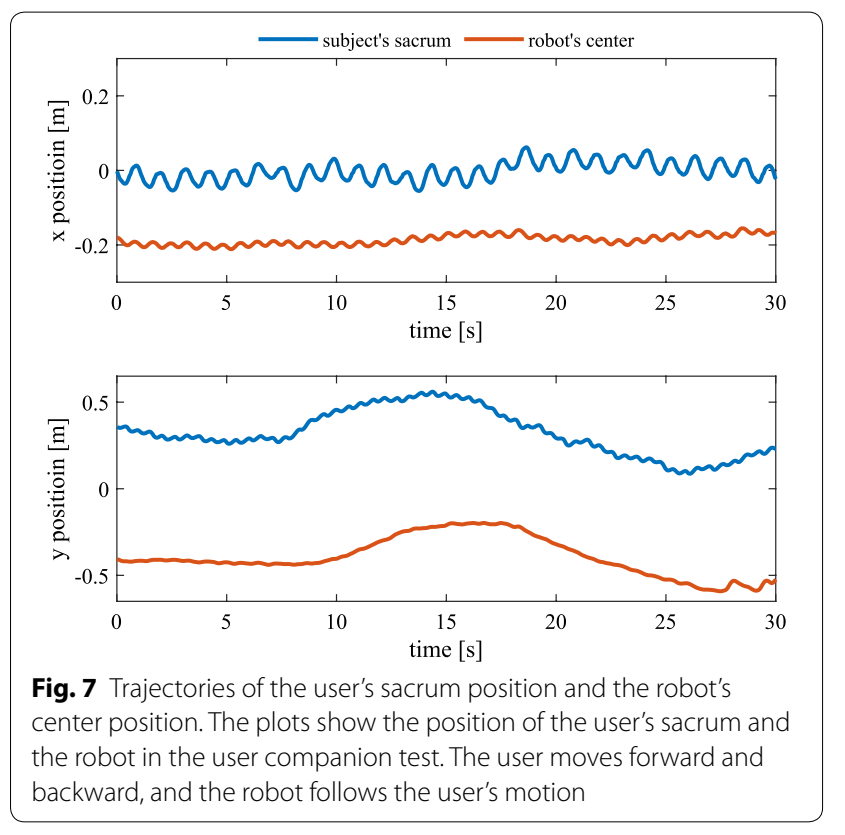

can be found in Fig. 7. The delay is caused by the PID and PD controllers in Eqs. (1) and (3). The delay possibly provide unsuitable interaction force to the subject. In future work, we will address a suitable control scheme to minimize such a time delay and a phase difference. In the clinical experiment mentioned below, we assume that the user's walking location on the treadmill is static or quasistatic and the control time delay does not affect the postural sway. From these results, the cane robot appears to be following the walking user appropriately and would be useful for practical use as a gait assistive device.

\section{Clinical experiment}

In this section, we present an experiment where subjects walk on a treadmill with the cane robot and we evaluate the posture stabilization effect by touching the robot. We introduce an experimental condition and conduct a clinical experiment using the treadmill used in the user companion test mentioned above. Then, we analyze the experimental result and discuss the potential of our strategy for the postural sway reduction using our cane robot.

\section{Condition}

In order to demonstrate proof of our concept of the posture stabilization effect by touching our cane robot on the treadmill, we conducted an experiment with three healthy subjects as a pilot study. The descriptive characteristics of the subjects are shown in Table 1. The subjects are instructed to walk on the treadmill at a constant speed of $0.8 \mathrm{~m} / \mathrm{s}$, which is one of the criteria for sarcopenia [37], for a duration of $30 \mathrm{~s}$. We compare 


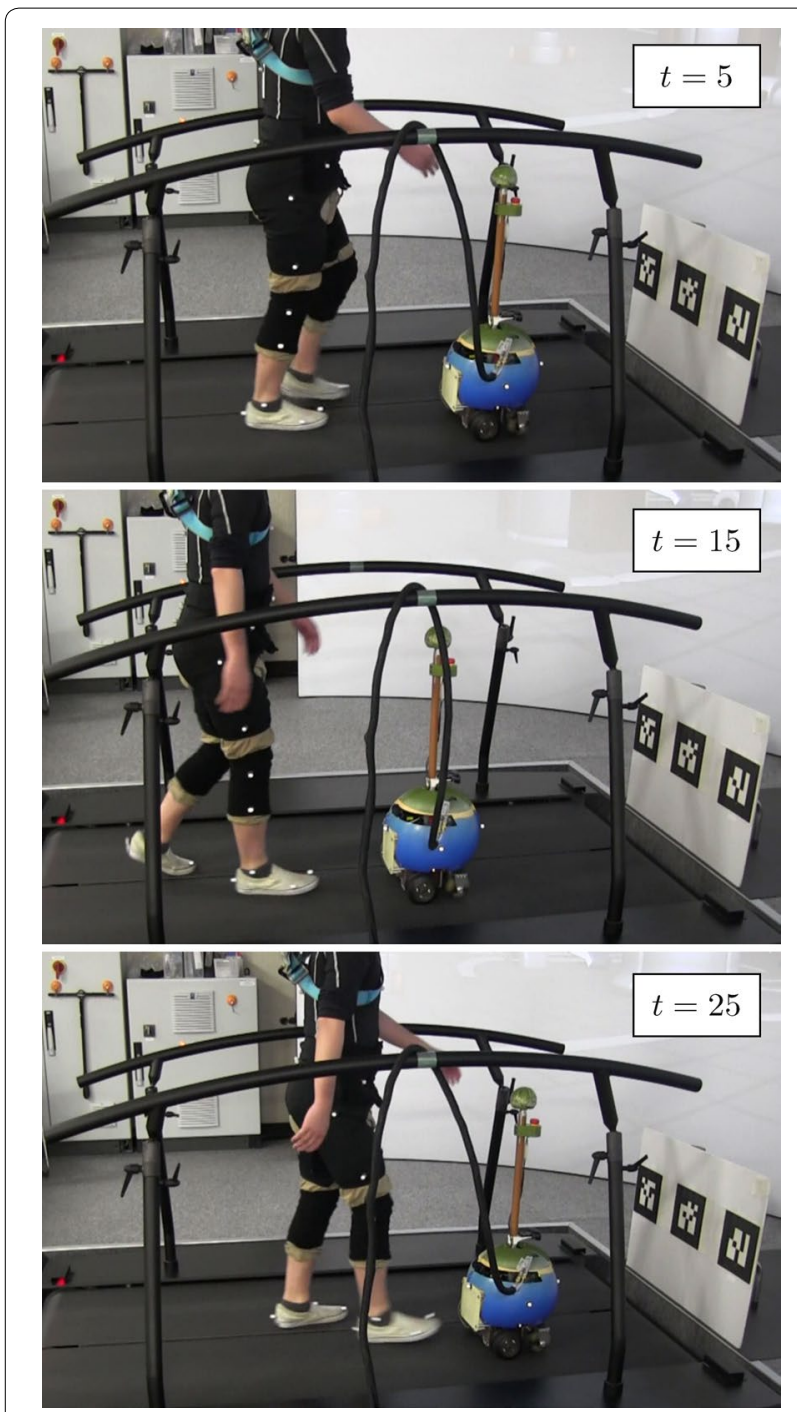

Fig. 8 Snapshots in the user companion test. A user is walking without touching the cane robot for $30 \mathrm{~s}$. These snapshots show the user and robot 5, 15, $25 \mathrm{~s}$ after the beginning of the test

two conditions where the subjects walk (1) without touching the cane robot (normal gait) and (2) with slightly touching the robot considering LTC mentioned in "Introduction" (assisted gait). The order of the trials is defined randomly. At the beginning of the trials, the subjects are allowed to change the cane robot position using the proposed manual control method. Then the cane robot moves to keep the configured relative position using the proposed autonomous tracking method. To ensure safety during the treadmill walking trial, the subjects wear harness-vest with straps. This experiment has been approved by the ethics committee of the National Center for Geriatrics and Gerontology in Japan.

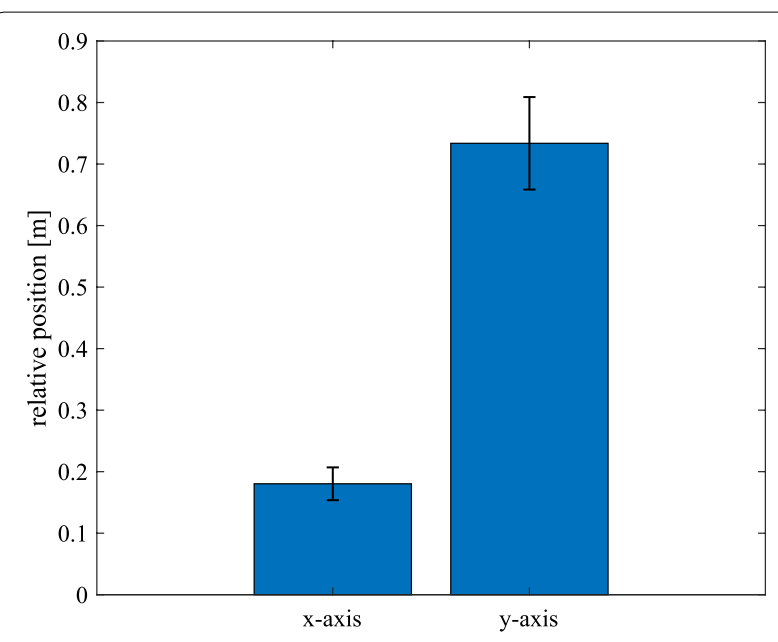

Fig. 9 Relative distance between the user and the robot in the user companion test. Although the user moved appropriately $0.5 \mathrm{~m}$ in $y$-axis in the test, the relative distance was almost constant and its standard division was about $0.075 \mathrm{~m}$

Table 1 Descriptive characteristics of the subjects in the experiment (values are given in mean \pm SD)

\begin{tabular}{lc}
\hline Age (years) & Gender \\
\hline $27.6 \pm 6$ & $M 1 / F 2$ \\
\hline
\end{tabular}

As the evaluation indices, in this paper, we focus on the oscillations of the sacrum position and tenth thoracic vertebrae (T10) position during gait trials in each condition (see Fig. 6). Note that, considering bipedal gait characteristics, it is obvious that the oscillation in y-axis and $\mathrm{x}$-axis would be affected by each gait cycle motion (two step cycles) and each step cycle motion, respectively. We apply the fast Fourier transform (FFT) analysis to the oscillated motions and compare the two conditions in terms of the amplitude and harmonic ratio at around the gait cycle frequency in y-axis and around the twofold frequency of the gait cycle in $x$-axis. The harmonic ratio is known as quantification of oscillation symmetry and often used to evaluate gait stability using acceleration data measured by an IMU as a simplified way in the physiological field [38]. In this paper, we apply this index to the measured position data measured by the motion capture system and discuss the user's stability.

\section{Results and discussion}

Figure 10 shows snapshots of the experimental setting in each condition: (1) without touching the cane robot (left) and (2) with touching the robot (right).

Figure 11 illustrates the mean and the standard division of gait cycle duration calculated from the motion data of 


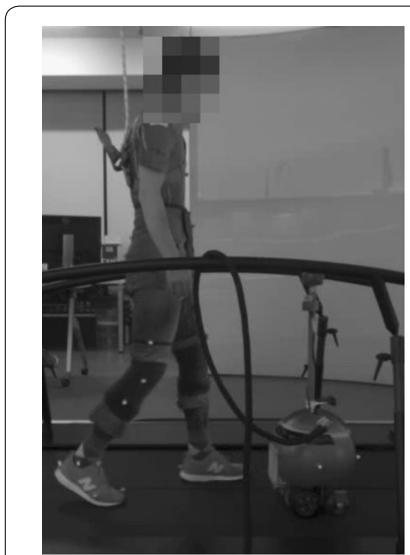

w/o touch

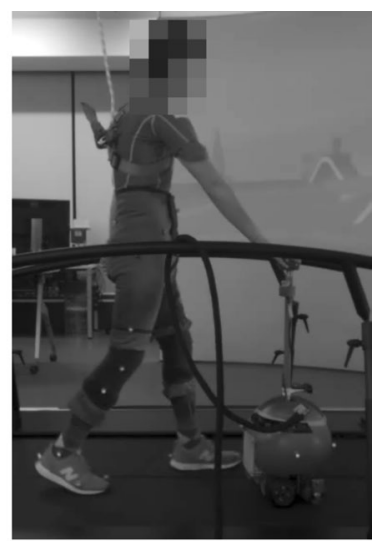

w/ touch
Fig. 10 Snapshots in the experiment. A subject is walking without touching (left) and with touching the cane robot with the right hand (right)

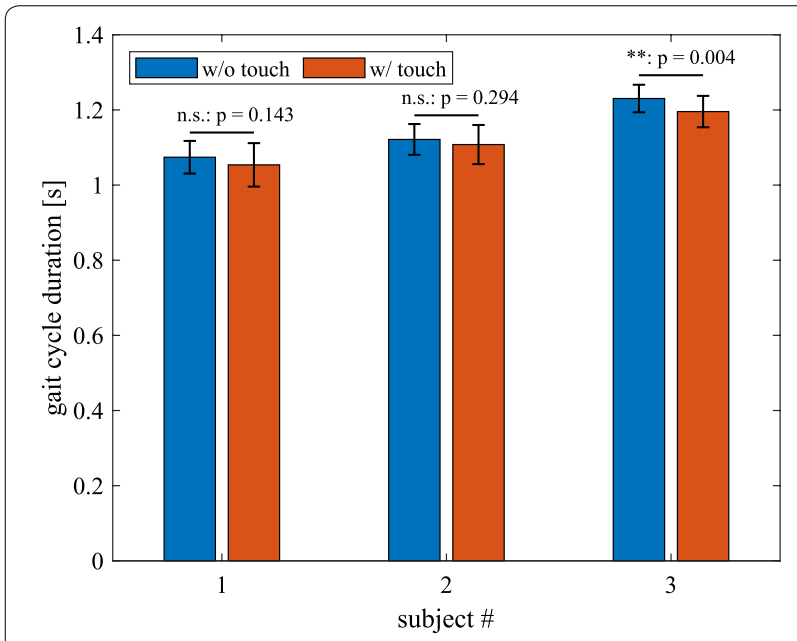

Fig. 11 Duration of the gait cycle of each subject. This graph shows the mean and standard deviation of the gait cycle durations. We found no significance in two subjects, although the step cycle of one of the subjects was shortened significantly. The step cycle is proportional to the step length since the speed of the treadmill belt is constant in this experiment

the subjects' heel during walking. Although the gait cycle duration of one subject was reduced significantly, we found no significance in the ones of the other subjects. The gait cycle duration is proportional to the step length since the walking speed is constant $0.8 \mathrm{~m} / \mathrm{s}$ in this experiment. The result suggests that the appearance of our cane robot may cause some change of gait posture or disturb some users to swing the leg physically or psychologically. For further investigation of postural sway reduction by light touch in future work, we will address to eliminate

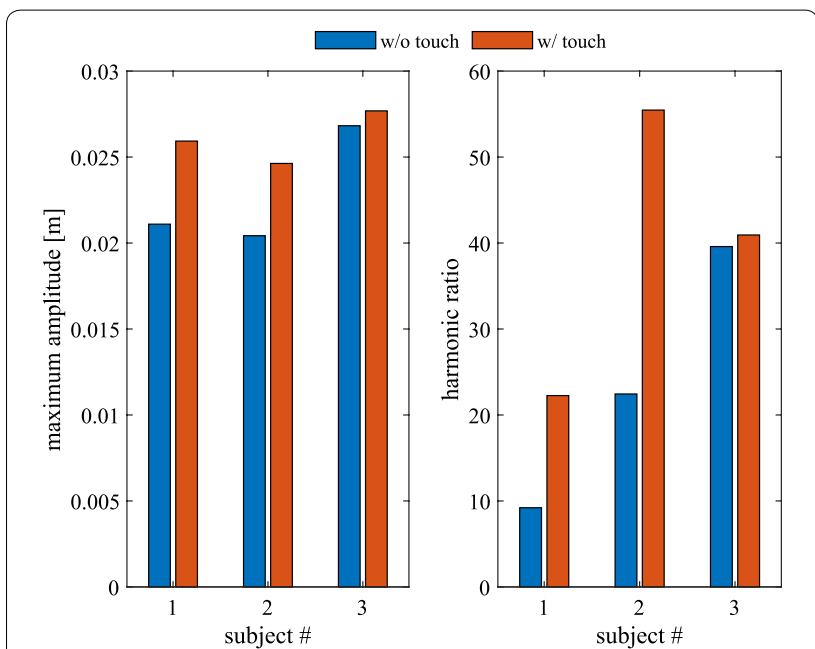

Fig. 12 Maximum amplitude and harmonic ratios of the sacrum positions of each subject in $x$-axis. The left figure shows the maximum amplitudes of the subjects'sacrum at the frequencies of the trials, and the right one shows their harmonic ratio in $x$-axis (lateral direction). The maximum amplitudes of no subject decreased. On the other hand, the harmonic ratios of all subjects increased

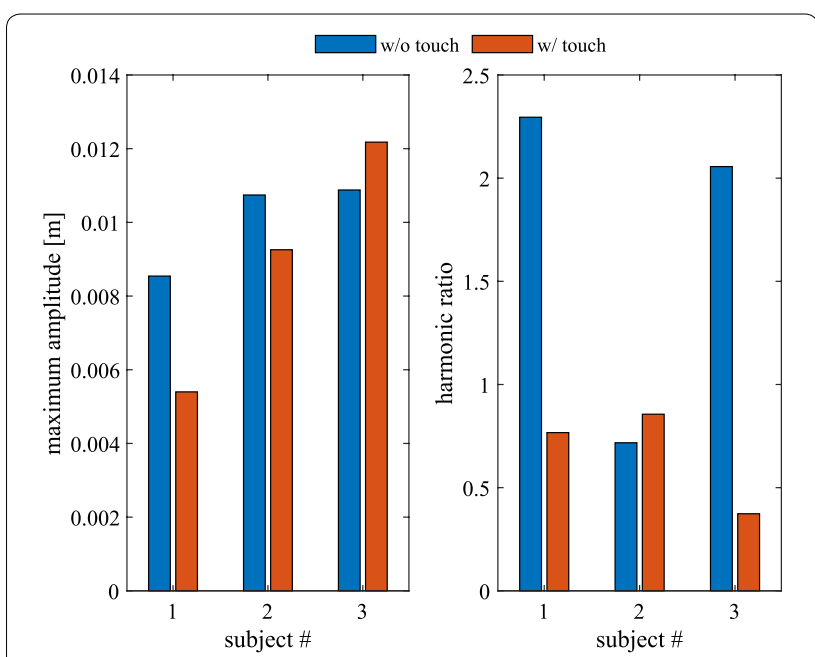

Fig. 13 Maximum amplitude and harmonic ratios of the sacrum positions of each subject in $y$-axis. The left figure shows the maximum amplitudes of the subjects' sacrum at the frequencies of the trials, and the right one shows their harmonic ratio in y-axis (frontal direction). The maximum amplitudes of the two subjects decreased. On the other hand, the harmonic ratio of one subject slightly increased

the effect on the swing leg, e.g., by adding a spring mechanism to absorb unexpected shock and limit the amount of interactive force. In the following evaluation, we focus on the postural sway of the subjects at frequencies of each subjects' gait cycle.

Figures 12 and 13 show the maximum amplitudes of the sacrum oscillation at the frequency of the gait and 
the harmonic ratios. The maximum amplitudes in $\mathrm{x}$-axis increased by $15.6 \pm 8.8 \%$, and the one in y-axis decreased by $12.9 \pm 19.9 \%$. In addition, their harmonic ratio in $\mathrm{x}$-axis increased by $97.4 \pm 66.5 \%$, and the one in y-axis decreased by $43.0 \pm 44.5 \%$. These results suggest that the touching motion and the obtained somatosensory feedback could provide useful restriction for stabilization of body sway. However, we could not find any cases that the touching condition provides positive effects on both of the evaluated indices.

Figures 14 and 15 illustrate the orientation angle of the subjects' posture calculated from a vector from their sacrum positions to the T10 positions projected on the coronal $(\mathrm{x}-\mathrm{z})$ and the sagittal $(\mathrm{y}-\mathrm{z})$ planes, respectively. The maximum amplitude of the oscillation of the angle projected on the coronal plane decreased by $24.9 \pm 20.4 \%$, and the one projected on the sagittal plane decreased by $22.9 \pm 11.3 \%$. Besides, their harmonic ratio on the coronal plane increased by $6.8 \pm 36.6 \%$, and the one on the sagittal plane decreased by $63.4 \pm 18.3 \%$. These results indicate that the touching action could help the subjects stabilize their body on the coronal plane. In terms of oriental oscillation on sagittal, the touching motion provides a positive effect on the maximum amplitude and the harmonic ratio both.

Note that this experiment was conducted with three healthy subjects having no disability, and it would be insufficient to conclude the assistive effectiveness of our research concept with statistical evaluation. We

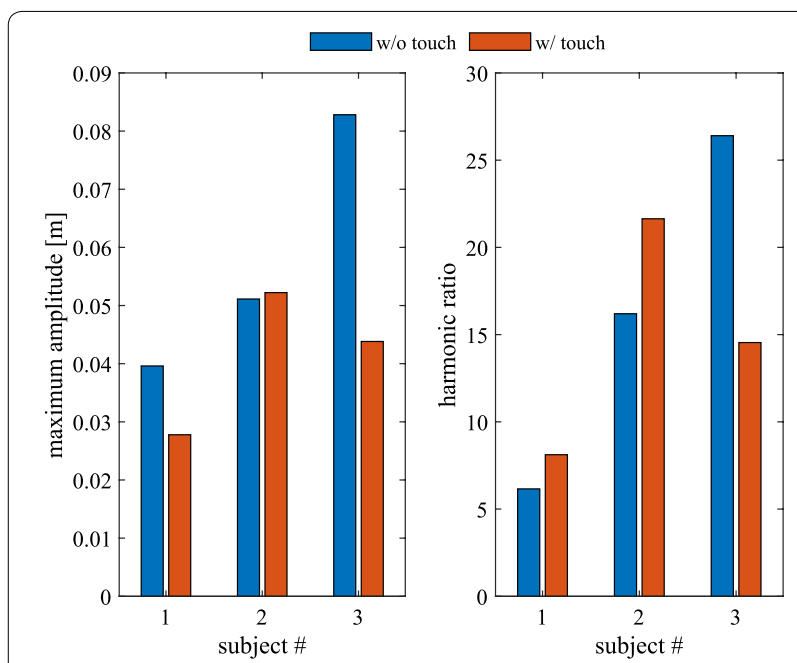

Fig. 14 Maximum amplitude and harmonic ratios of the postural angle projected on the coronal plane of each subject. The left figure shows the maximum amplitudes of the postural angle projected on the coronal plane at the frequencies of the trials, and the right figure shows the harmonic ratios of them. The maximum amplitudes of the two subjects slightly decreased. On the other hand, the harmonic ratios of two of the subjects increased

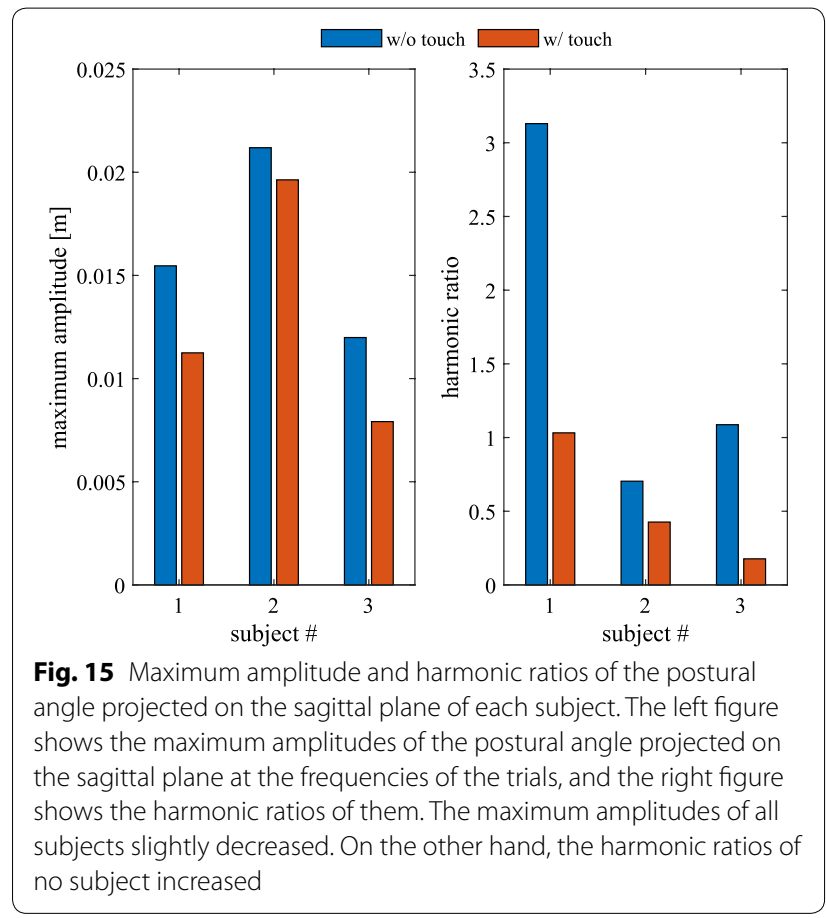

demonstrate proof of our concept on our cane-type companion robot for gait stabilization through the experimental result.

The absolute vertical force applied to the cane robot by the user walking with touching the robot is shown in Fig. 16. Although the subjects were instructed to walk

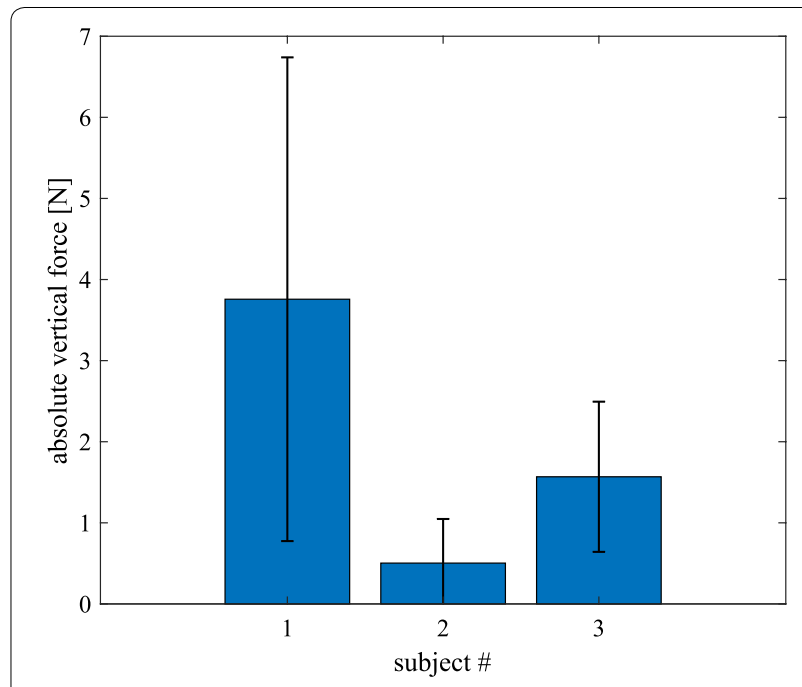

Fig. 16 Absolute vertical force of the subjects applied to the robot handle during walking. The plots show the absolute vertical force $\left|f_{z}\right|$ applied to the cane robot by the subjects walking on the treadmill. Before the experiments, the subjects were instructed to touch the robot slightly 
with lightly touching the robot, the maximum applied force changed approximately $10 \mathrm{~N}$ between the subjects. The average of the force was $1.94 \pm 2.28 \mathrm{~N}$, and the LTC effect, which is human stabilization functionality mentioned in "Introduction", could affect the above experimental results. We will address to investigate the LTC promoted by our cane robot in future work.

\section{Conclusion}

In this paper, we proposed a user companion algorithm for our cane robot moving on the treadmill with the user in order to investigate the postural sway reduction by slightly touching the robot. To solve the problems peculiar to the gait trial on the treadmill, the AR markers are placed in front of the robot and used as a landmark for the self-location. Then, we introduced a manual and autonomous motion planning methodologies according to the applied force from the user and the relative position between the robot and the user, respectively. Through a simple walking test with one user, we evaluated the accuracy of the user companion achieved by the proposed autonomous motion planning. In addition, we conducted an experiment to investigate the touching effect with three healthy subjects walking on the treadmill with our cane robot as a pilot study, and evaluated the amplitude and the harmonic ratio of the sway. Through the experiments, we demonstrated proof of concept of our walking assistance strategy using the cane-type companion robot.

As mentioned, we could not conclude the effectiveness of our robotic stabilization approach with statistical evaluation. In future work, we will conduct experiments with more number of healthy and elderly people, and compare various conditions such as faster/slower walking speed to confirm the effectiveness of the proposed walking assistance. In addition, we will address redesigning the cane robot hardware not to disturb the user's leg swing as well as motion control algorithm to enable the robot to accompany the user walking around on a flat floor.

\section{Abbreviations \\ QOL: quality of life; LTC: light touch contact; LRF: laser range finder; IMU: inertial measurement unit; ROS: robot operating system; GUI: graphical user interface; FFT: fast Fourier transform.}

\section{Acknowlegements}

This research was funded in part by the Research Funding for Longevity Sciences (28-34) form the National Center for Geriatrics and Gerontology (NCGG).

\section{Authors' contributions}

$\mathrm{SI}, \mathrm{TA}$, and $\mathrm{YH}$ developed the cane robot and designed the control algorithm. $\mathrm{KA}, \mathrm{KK}$, and IK designed and conducted the experiment. SI and KA analyzed and interpreted the data, and drafted the manuscript. All authors read and approved the final manuscript.
Availability of data and materials

Please contact author for data requests.

\section{Ethics approval and consent to participate}

The study protocol was approved by the National Center for Geriatrics and Gerontology and was carried out in accordance with their recommendations. All subjects were given a written informed consent in accordance with the Declaration of Helsinki.

\section{Consent for publication}

Not applicable.

\section{Competing interests}

The authors declare that they have no competing interests.

\section{Author details}

${ }^{1}$ Department of Micro-Nano Mechanical Science and Engineering, Nagoya University, Nagoya 464-8603, Japan. ${ }^{2}$ Department of Rehabilitation, National Center for Geriatrics and Gerontology, Obu 474-8511, Japan.

Received: 23 August 2019 Accepted: 16 November 2019

Published online: 27 November 2019

\section{References}

1. United Nations (2001) World Population Ageing: 1950-2050. http://www. un.org/esa/population/publications/worldageing19502050

2. Proot IM, Abu-Saad HH, Crebolder HFJM, Goldsteen M, Luker KA, Widdershoven GAM (2003) Vulnerability of family caregivers in terminal palliative care at home; balancing between burden and capacity. Scand J Caring Sci 17(2):113-121

3. Kondo I (2019) Frailty in an aging society and the applications of robots. Jpn J Compr Rehabil Sci 10:47-49

4. Bortz WM (1984) The disuse syndrome. Western J Med 141(5):691-694

5. Fraga MJ, Cader SA, Ferreira MA, Giani TS, Dantas EHM (2011) Aerobic resistance, functional autonomy and quality of life (QoL) of elderly women impacted by a recreation and walking program. Arch Gerontol Geriatr 52(1):40-43

6. Viteckova S, Kutilek P, Jirina M (2013) Wearable lower limb robotics: a review. Biocybern Biomed Eng 33(2):96-105

7. Chen B, Ma H, Qin LY, Gao F, Chan KM, Law SW et al (2016) Recent developments and challenges of lower extremity exoskeletons. J Orthop Transl 5:26-37

8. Esclarín-Ruz A, Alcobendas-Maestro M, Casado-Lopez R, Perez-Mateos G, Florido-Sanchez MA, Gonzalez-Valdizan E et al (2014) A comparison of robotic walking therapy and conventional walking therapy in individuals with upper versus lower motor neuron lesions: a randomized controlled trial. Arch Phys Med Rehabil 95(6):1023-1031

9. Capristo E, Malandrino N, Farnetti S, Mingrone G, Leggio L, Addolorato $\mathrm{G}$ et al (2009) Increased serum high-density lipoprotein-cholesterol concentration in celiac disease after gluten-free diet treatment correlates with body fat stores. J Clin Gastroenterol 43(10):946-949

10. Evans N, Hartigan C, Kandilakis C, Pharo E, Clesson I (2015) Acute cardiorespiratory and metabolic responses during exoskeleton-assisted walking overground among persons with chronic spinal cord injury. Topics Spinal Cord Injury Rehabil 21(2):122-132

11. Kawamoto H, Kamibayashi K, Nakata Y, Yamawaki K, Ariyasu R, Sankai Y et al (2013) Pilot study of locomotion improvement using hybrid assistive limb in chronic stroke patients. BMC Neurol 13:141

12. Tanabe S, Hirano S, Saitoh E (2013) Wearable Power-Assist Locomotor (WPAL) for supporting upright walking in persons with paraplegia. Neuro Rehabil 33(1):99-106

13. Mori Y, Okada J, Takayama K (2004) Development of straight style transfer equipment for lower limbs disabled. In: IEEE international conference on robotics and automation. pp 2486-2491

14. Kong K, Jeon D (2006) Design and control of an exoskeleton for the elderly and patients. IEEE/ASME Trans Mech 11(4):428-432 
15. Ikumi A, Kubota S, Shimizu Y, Kadone H, Marushima A, Ueno T et al (2017) Decrease of spasticity after hybrid assistive limb training for a patient with C4 quadriplegia due to chronic SCI. J Spinal Cord Med 40(5):573-578

16. Kikuchi T, Tanaka T, Anzai K, Kawakami S, Hosaka M, Niino K (2013) Evaluation of line-tracing controller of intelligently controllable walker. Adv Robot 27(7):493-502

17. Hirata Y, Hara A, Kosuge K (2007) Motion control of passive intelligent walker using servo brakes. IEEE Trans Robot 23(5):981-990

18. Spenko M, Yu H, Dubowsky S (2006) Robotic personal aids for mobility and monitoring for the elderly. IEEE Trans Neural Syst Rehabil Eng 14(3):344-351

19. Jeka J (1997) Light touch contact as a balance aid. Phys Ther 77(5):476-487

20. Jeka JJ, Lackner JR (1994) Fingertip contact influences human postural control. Exp Brain Res 79(2):495-502

21. Jeka JJ, Lackner JR (1995) The role of haptic cues from rough and slippery surfaces in human postural control. Exp Brain Res 103(2):267-276

22. Holden M, Ventura J, Lackner JR (1994) Stabilization of posture by precision contact of the index finger. J Vestib Res 4(4):285-301

23. Johannsen L, Wing AM, Hatzitaki V (2007) Effects of maintaining touch contact on predictive and reactive balance. J Neurophysiol 97(4):26862695. https://doi.org/10.1152/jn.00038.2007

24. Lackner JR, DiZio P, Jeka J, Horak F, Krebs D, Rabin E (1999) Precision contact of the fingertip reduces postural sway of individuals with bilateral vestibular loss. Exp Brain Res 126(4):459-466

25. Dickstein R, Shupert CL, Horak FB (2001) Fingertip touch improves postural stability in patients with peripheral neuropathy. Gait Posture 14(3):238-247

26. Boonsinsukh R, Panichareon L, Phansuwan-Pujito P (2009) Light touch cue through a cane improves pelvic stability during walking in stroke. Arch Phys Med Rehabil 90(6):919-926. https://doi.org/10.1016/j. apmr.2008.12.022

27. Dickstein R, Laufer $Y$ (2004) Light touch and center of mass stability during treadmill locomotion. Gait Posture 20(1):41-47

28. Shima K, Shimatani K, Sugie A, Kurita Y, Kohno R, Tsuji T (2013) Virtua light touch contact: a novel concept for mitigation of body sway. In: International symposium on medical information and communication technology, ISMICT. pp 108-111
29. Di P, Hasegawa Y, Nakagawa S, Sekiyama K, Fukuda T, Huang J et al (2016) Fall detection and prevention control using walking aid cane robot. IEEE/ ASME Trans Mech 21(2):625-637

30. Itadera S, Hasegawa Y, Fukuda T, Tanimoto M, Kondo I (2017) Adaptive walking load control for training physical strength using cane-type robot. In: IEEE/RSJ international conference on intelligent robots and systems. pp 521-526

31. Nakagawa S, Hasegawa Y, Fukuda T, Kondo I, Tanimoto M, Di P et al (2016) Tandem stance avoidance using adaptive and asymmetric admittance control for fall prevention. IEEE Trans Neural Syst Rehabil Eng 24(5):542-550

32. Quigley M, Gerkey B, Conley K, Faust J, Foote T, Leibs J, et al (2009) ROS: an open-source robot operating system. In: IEEE international conference on robotics and automation, open-source software workshop

33. Bohannon RW (1997) Comfortable and maximum walking speed of adults aged 20-79 years: reference values and determinants. Age Ageing 26(1):15-19

34. Wada M, Takagi A, Mori S (2000) Caster drive mechanisms for holonomic and omnidirectional mobile platforms with no over constraint. In: IEEE international conference on robotics and automation. pp 1531-1538

35. Lu DV, Smart WD (2013) Towards more efficient navigation for robots and humans. In: IEEE international conference on intelligent robots and systems. pp 1707-1713

36. Motekforce. GRAIL. https://www.motekforcelink.com/product/grail/

37. Cruz-Jentoft AJ, Baeyens JP, Bauer JM, Boirie Y, Cederholm T, Landi F et al (2010) Sarcopenia: European consensus on definition and diagnosis. Age Ageing 39(4):412-423

38. Roche JL, Lowry KA, Vanswearingen JM, Brach JS, Redfern MS, Division G (2016) Harmonic ratios: a quantification of step to step symmetry. J Biomech 46(4):828-831

\section{Publisher's Note}

Springer Nature remains neutral with regard to jurisdictional claims in published maps and institutional affiliations.

\section{Submit your manuscript to a SpringerOpen ${ }^{\circ}$ journal and benefit from:}

- Convenient online submission

- Rigorous peer review

- Open access: articles freely available online

- High visibility within the field

- Retaining the copyright to your article

Submit your next manuscript at $\boldsymbol{\nabla}$ springeropen.com 